\title{
Summary of clinically diagnosed amniotic fluid embolism cases in Korea and disagreement with 4 criteria proposed for research purpose
}

\author{
Jin-ha Kim, MD ${ }^{1}$, Hyun-Joo Seol, MD, PhD², Won Joon Seong, MD, PhD ${ }^{3}$, Hyun-Mee Ryu, MD, PhD ${ }^{4}$, \\ Jin-Gon Bae, MD, PhD ${ }^{5}$, Joon Seok Hong, MD, PhD ${ }^{6}$, Jeong In Yang, MD, PhD ${ }^{7}$ Ji-Hee Sung, MD, PhD ${ }^{1}$, \\ Suk-Joo Choi, MD, PhD ${ }^{1}$, Soo-young Oh, MD, PhD ${ }^{1}$, Cheong-Rae Roh, MD, PhD ${ }^{1}$
}

\begin{abstract}
Department of Obstetrics and Gynecology, ${ }^{1}$ Samsung Medical Center, Sungkyunkwan University School of Medicine, ${ }^{2}$ Kyung Hee University School of Medicine, Seoul; ${ }^{3}$ Kyungpook National University Hospital, Daegu; ${ }^{4} \mathrm{CHA}$ Bundang Medical Center, CHA University School of Medicine, Seongnam; ${ }^{5}$ Keimyung University School of Medicine, Daegu; ' ${ }^{6}$ Seoul National University Bundang Hospital, Seongnam; ${ }^{7}$ Ajou University School of Medicine, Suwon, Korea
\end{abstract}

\section{Objective}

This study aimed 1) to investigate the clinical characteristics of amniotic fluid embolism (AFE) cases clinically diagnosed by maternal fetal medicine (MFM) specialists in Korea, 2) to check the disagreement with 4 recently proposed criteria by the Society for Maternal-Fetal Medicine (SMFM) for research purpose, and 3) to compare maternal outcomes between cases satisfying all 4 criteria and cases with at least 1 missing criterion.

Methods

This study included 12 patients clinically diagnosed with AFE from 7 referral hospitals in Korea. We collected information, including maternal age, symptoms of AFE, the amount of transfusion, and maternal mortality.

\section{Results}

The median maternal age was 33 years (range, 28-40 years). Regarding symptoms, cardiovascular arrest, hypotension, respiratory compromise, clinical coagulopathy, and neurologic signs were observed in $41.7 \%, 83.3 \%, 83.3 \%, 100 \%$, and $66.7 \%$ of the cases, respectively. Among the 12 cases, 5 women died and 2 suffered severe neurologic disability, showing an intact survival rate of $41.7 \%$. Disagreement with all 4 criteria proposed by the SMFM was found in $66.7 \%$ of the cases, due to the lack of criteria for disseminated intravascular coagulation or strict onset time $(<30$ minutes after delivery). There was no difference in maternal mortality and the amount of transfusion between cases satisfying all 4 criteria and cases with at least 1 missing criterion.

\section{Conclusion}

Two-thirds of clinically confirmed AFE cases did not satisfy all 4 criteria proposed by the SMFM, despite similar rates of maternal mortality with cases satisfying all 4 criteria. Our study suggests that there may be some discrepancy between the clinical diagnosis of AFE and the recent diagnostic criteria proposed by the SMFM for research purpose.

Keywords: Amniotic fluid embolism; Criteria; Diagnosis; Korea

Received: 2020.07.08. Revised: 2020.10.13. Accepted: 2020.10.21.

Corresponding author: Soo-young $\mathrm{Oh}, \mathrm{MD}, \mathrm{PhD}$

Department of Obstetrics and Gynecology, Samsung Medical

Center, Sungkyunkwan University School of

Medicine, 81 Irwon-ro, Gangnam-gu, Seoul 06351, Korea

E-mail: ohsymd@skku.edu

https://orcid.org/0000-0003-3002-0048
Cheong-Rae Roh has been an Editorial Board of Obstetrics \& Gynecology Science; however, she(he) was not involved in the peer reviewer selection, evaluation, or decision process of this article. Otherwise, no other potential conflicts of interest relevant to this article was reported.

Articles published in Obstet Gynecol Sci are open-access, distributed under the terms of the Creative Commons Attribution Non-Commercial License (http://creativecommons. org/licenses/by-nc/3.0/) which permits unrestricted non-commercial use, distribution, and reproduction in any medium, provided the original work is properly cited.

Copyright (c) 2020 Korean Society of Obstetrics and Gynecology 


\section{Obstetrics \& Gynecology Science}

Jin-ha Kim, et al. Diagnosis of amniotic fluid embolism

\section{Introduction}

Amniotic fluid embolism (AFE) is a rare and unpredictable complication of pregnancy that appears during delivery or in the immediate postpartum period. Despite its unusual occurrence of 1 in 10,000 to 50,000 deliveries, AFE is one of the leading causes of maternal mortality, estimated to be a causative factor in about $20-60 \%$ of maternal mortality rates globally $[1,2]$. According to the most recent statistics from Korea, obstetric embolism, including AFE and pulmonary embolism, accounted for $21.6 \%$ of direct maternal deaths in 2018 [3].

In general, AFE is clinically diagnosed as sudden cardiovascular collapse, after exclusion of other possible causes. Several countries have provided diagnostic criteria for AFE, which are not in complete agreement with each other. For example, the diagnostic criteria in Australia are based on "clinical diagnosis, including acute hypotension or cardiac arrest, acute hypoxia, or coagulopathy in the absence of any other potential explanation for signs and symptoms observed or pathologic diagnosis of fetal squamous cells or hair in lungs" [4]. In the United Kingdom, AFE is defined as follows. "In the absence of any other clear cause, either 1) acute maternal collapse with one or more of the following features: acute fetal compromise, cardiac arrhythmias, etc., 2) excluding women with maternal hemorrhage as the first presenting feature, in whom there was no evidence of early coagulopathy or cardiorespiratory compromise; or 3) women in whom the diagnosis was established during post-mortem examination by finding fetal squamous cells or hair in the lungs" [5]. In Japan, AFE is defined if 1) symptoms appeared during pregnancy or within 12 hours of delivery; 2 ) intensive medical intervention was conducted to treat $\geq 1$ of the following symptoms/diseases: (a) cardiac arrest, (b) severe bleeding of unknown origin within 2 hours of delivery $(\geq 1,500 \mathrm{~mL}),(\mathrm{c})$ disseminated intravascular coagulation (DIC), or (d) respiratory failure; 3) findings or symptoms obtained could not be explained by other diseases; and 4) consumptive coagulopathy/ DIC due to evident etiologies, such as abnormal placentation, trauma during labor, and severe preeclampsia/eclampsia, was excluded [6].

Such multiple definitions of AFE from different countries hinder the identification of consistent risk factors of AFE, evaluation of the efficacy of treatments, and understanding of the exact pathophysiology of this notorious obstetric prob- lem. Thus, in 2016, the Society for Maternal-Fetal Medicine (SMFM) and the Amniotic Fluid Embolism Foundation proposed centralized diagnostic criteria for AFE [7]. According to their definition, there are 4 criteria that a case must meet to be diagnosed as AFE, which are as follows: 1) sudden onset of cardiorespiratory arrest, or both hypotension and respiratory compromise; 2) documentation of overt DIC after appearance of these initial signs or symptoms, where coagulopathy must be detected before loss of sufficient blood to account for dilutional or shock-related consumptive coagulopathy; 3) clinical onset during labor or within 30 minutes of delivery of the placenta; and 4) no fever during labor [7].

However, direct application of these criteria in real clinical circumstances has been questioned [8,9]. In fact, according to a French national study, including 36 women who died of AFE, $41.7 \%$ did not satisfy one or more diagnostic criteria presented by the SMFM. The most unsatisfied criterion was that the DIC finding should be present after amniotic embolism before a sufficient amount of blood loss. Accordingly, the authors suggested expanding the criteria of early documented DIC proposed by the SMFM, to early clinical coagulopathy with bleeding [8].

Since AFE is a major cause of uncontrollable and unpredictable maternal death, legal disputes are sometimes inevitable. Unfortunately, in Korean precedents, there seems to be a tendency not to recognize AFE as a cause of mortality, especially in cases involving residual placenta, birth canal laceration, and placental abruption [10-12], which are commonly combined with AFE as important risk factors $[13,14]$. Furthermore, the social reluctance to perform autopsy in Korea often aggravates the legal debate, relying more on clinical manifestations. Our authors, including several maternal fetal medicine (MFM) specialists in Korea, are concerned that the inadvertent presentation of the SMFM criteria to court may not recapitulate real clinical manifestations of AFE, resulting in misinterpretation of medico-legal judgments.

Given this background, the aim of this study was 1) to investigate the clinical characteristics of AFE cases diagnosed by MFM specialists in Korea, 2) to check the disagreement with the 4 criteria provided by the SMFM and the Amniotic Fluid Embolism Foundation, and 3) to compare maternal outcomes between cases satisfying all 4 criteria and those with at least 1 missing criterion. 


\title{
Obstetrics \& Gynecology Science
}

\author{
Vol. 64, No. 2, 2021
}

\section{Materials and methods}

This study included 12 patients clinically diagnosed with AFE by MFM specialists from 7 referral hospitals in Korea, including Samsung Medical Center, Kyung Hee University Hospital, Cheil Medical Center, Kyungpook National University Hospital, Keimyung University Hospital, Seoul National University Bundang Hospital, and Ajou University Hospital, from 2008 to 2019.

We collected information, including baseline maternal characteristics, delivery outcomes, and clinical manifestations of AFE, through the questionnaire survey (Supplementary Data 1). Baseline maternal characteristics included age, body mass index at the time of diagnosis of AFE, and gestational age at delivery. Some intrapartum factors were also recorded, including spontaneous labor, induction or augmentation (oxytocin use) of labor, and mode of delivery (spontaneous vaginal delivery, emergent cesarean section, elective cesarean section).

AFE symptoms were classified into 5 groups as follows: cardiovascular collapse (cardiac arrest), hypotension, respiratory compromise (dyspnea, coughing, cyanosis, shortness of breath), clinical coagulopathy, and neurological signs (restlessness, confusion, agitation, numbness, tingling, fainting, mental change, and seizure). Of these, we also identified the first manifested symptom. Massive obstetric hemorrhage was defined as a bleeding volume of $>1,000 \mathrm{~mL}$. Clinical coagulopathy was defined as an incoagulable maternal hemorrhage of unknown origin during the intrapartum or postpartum period. The laboratory diagnosis of DIC was based on platelet count, prothrombin time, and fibrinogen levels. The clinical onset time of these signs and symptoms was divided into antepartum (before the onset of labor), intrapartum (from the onset of labor to the delivery of the placenta), and postpartum (after the delivery of the placenta). We also examined variables associated with the management of obstetric hemorrhage, including the amount of transfusion (red blood cells, platelets, fresh frozen plasma, cryoprecipitate), extracorporeal membrane oxygenation (ECMO), hysterectomy, and intensive care unit (ICU) care. Maternal outcomes were also investigated for mortality and complications, such as neurologic sequelae. Neonatal data included sex, birthweight, Apgar score, survival, and hypoxic-ischemic encephalopathy.

We applied the definition proposed by the SMFM and the
Amniotic Fluid Embolism Foundation to each case of AFE and checked the disagreement. This definition is based on 4 diagnostic criteria, all of which should exist in any instance of AFE. The criteria are as follows: 1) sudden onset of cardiorespiratory arrest, or both hypotension (systolic blood pressure $<90 \mathrm{~mm} \mathrm{Hg}$ ) and respiratory compromise (dyspnea, cyanosis, or peripheral capillary oxygen saturation $<90 \%$ ), 2) documentation of overt DIC after appearance of these initial signs or symptoms using the scoring system of the Scientific and Standardization Committee of the International Society on Thrombosis and Hemostasis (ISTH) for DIC, modified for pregnancy. Coagulopathy must be detected before loss of sufficient blood to account for dilutional or shockrelated consumptive coagulopathy; 3 ) clinical onset during labor or within 30 minutes of the delivery of the placenta; and 4) no fever (temperature $\geq 38^{\circ} \mathrm{C}$ ) during labor. Accord-

Table 1. Clinical features of mode of delivery, type of labor, symptoms, and clinical onset time of amniotic fluid embolism (AFE) cases

\begin{tabular}{lc}
\hline Clinical features & Total ( $\mathbf{n = 1 2 )}$ \\
\hline Mode of delivery & $6(50.0)$ \\
Spontaneous vaginal delivery & $4(33.3)$ \\
Cesarean section with labor & $2(16.7)$ \\
Cesarean section without labor & 9 a) \\
Type of labor & $4(44.4)$ \\
Spontaneous labor & $3(33.3)$ \\
Induction of labor & $2(22.2)$ \\
Augmentation of labor & \\
Symptoms & $5(41.7)$ \\
Cardiovascular arrest & $10(83.3)$ \\
Hypotension & $10(83.3)$ \\
Respiratory compromise & $12(100.0)$ \\
Clinical coagulopathy & $8(66.7)$ \\
Neurologic signs & \\
Clinical onset time & $0(0.0)$ \\
Antepartum & $5(41.7)$ \\
Intrapartum & $7(58.3)$ \\
Postpartum & $2 / 7(28.6)$ \\
$<30$ minutes after delivery & \\
1-2 hours after delivery & as a denominator. \\
\hline the labor type was not assessed, finally being & \\
\hline &
\end{tabular}




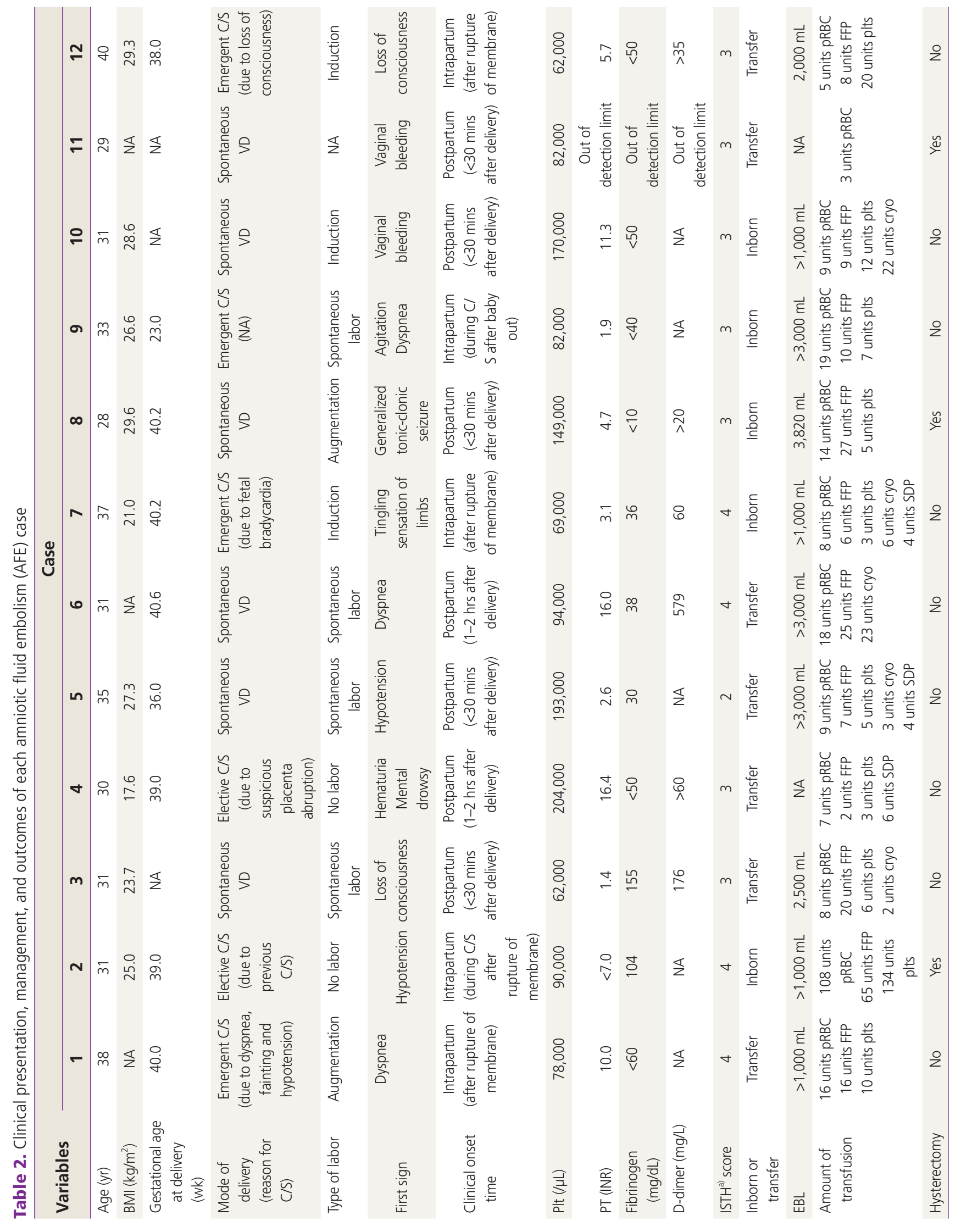




\section{Obstetrics \& Gynecology Science}

Vol. 64, No. 2, 2021

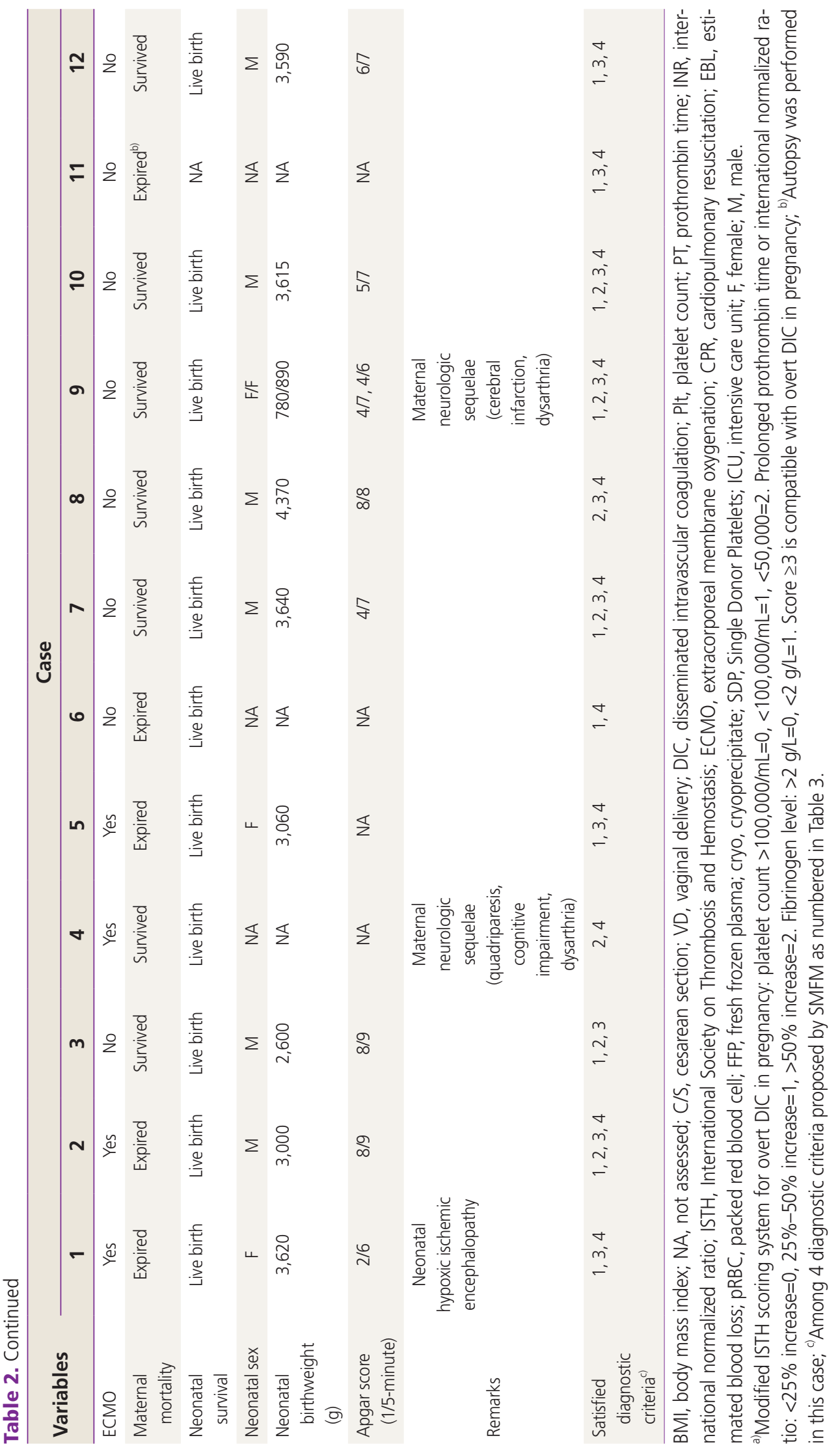




\section{Obstetrics \& Gynecology Science}

Jin-ha Kim, et al. Diagnosis of amniotic fluid embolism

ing to the modified ISTH scoring system for overt DIC in pregnancy, a score $\geq 3$ is compatible with DIC in pregnancy, with platelet count $>100,000 / \mathrm{mL}=0,<100,000 / \mathrm{mL}=1$, and $<50,000 \mathrm{~mL}=2$; prolonged prothrombin time or international normalized ratio, $<25 \%$ increase $=0,25 \%-50 \%$ increase $=1$, and $>50 \%$ increase $=2$; and fibrinogen level $>2 \mathrm{~g} / \mathrm{L}=0$ and $<2 \mathrm{~g} / \mathrm{L}=1$ [7].

We divided the study population into the cases that satisfied all 4 criteria and those that did not, and compared the mortality rate, massive hemorrhage, and intensive care (amount of transfusion, ECMO, hysterectomy, and ICU care) using the Mann-Whitney $\mathrm{U}$ test and Fisher exact test.

\section{Results}

This study included 12 cases clinically diagnosed with AFE. Seven of these cases were transferred from local hospitals to referral hospitals, and 5 cases delivered in referral hospitals. The median maternal age was 33 years (range 28-40 years).

Table 1 summarizes the delivery characteristics, clinical symptoms, and onset time of the 12 AFE cases. Ten patients experienced labor, while 2 patients underwent cesarean section without labor. Among the cases with labor, spontaneous labor, induction of labor, and augmentation of labor were noted in $44.4 \%, 33.3 \%$, and $22.2 \%$ cases, respectively. Re- garding AFE symptoms, cardiovascular arrest, hypotension, respiratory compromise, clinical coagulopathy, and neurologic signs were observed in $41.7 \%, 83.3 \%, 83.3 \%, 100 \%$, and $66.7 \%$ of the cases, respectively. The clinical onset time of the signs and symptoms was antepartum, intrapartum, and postpartum in $0 \%, 41.7 \%$, and $58.3 \%$ of the cases, respectively. Within the postpartum period, $71.4 \%$ of the cases developed symptoms within 30 minutes of delivery, and 28.6\% developed symptoms between 1 and 2 hours after delivery.

Table 2 summarizes the clinical presentation, management, and outcomes of AFE cases. As a first sign, neurologic signs, such as loss of consciousness, mental change, tingling sensation, seizure, and agitation, were the most common (50\%), followed by dyspnea (25\%), hypotension (16.7\%), and vaginal bleeding (16.7\%). The median amount of transfusion was 9 red blood cell (RBC), 5.5 platelet, and 9.5 FFP packs. The maximum amount of transfusion was 108 RBC, 134 platelet, and 65 FFP packs, a total of 307 packs in case number 2, who eventually expired.

Massive obstetric hemorrhage $(>1,000 \mathrm{~mL})$ occurred in 9 cases, and the estimated blood loss was not documented in 2 cases. Among 12 cases, hysterectomy and ECMO were performed in 3 (25.0\%) and 4 (33.3\%), respectively. Regarding maternal mortality, 5 (41.7\%) women died and 2 (16.7\%) women suffered severe neurologic disability, showing that in-

Table 3. Disagreement between amniotic fluid embolism (AFE) cases and 4 criteria proposed by Society for Maternal-Fetal Medicine (SMFM)

\begin{tabular}{|c|c|c|}
\hline \multirow{2}{*}{ Diagnostic criteria for AFE study } & \multicolumn{2}{|c|}{ Total $(n=12)$} \\
\hline & Yes (\%) & No (\%) \\
\hline All 4 diagnostic criteria & $4(33.3)$ & $8(66.7)$ \\
\hline $\begin{array}{l}\text { 1. Sudden onset of cardiorespiratory arrest or both hypotension (SABP }<90 \mathrm{mmHg} \text { ) and respiratory } \\
\text { compromise (dyspnea, cyanosis, } \mathrm{SpO} 2<90 \% \text { ) }\end{array}$ & $10(83.3)$ & $2(16.7)$ \\
\hline $\begin{array}{l}\text { 2. Documentation of overt DIC after appearance of these initial signs or symptoms, using scoring } \\
\text { system of Scientific and Standardization Committee on DIC of the ISTH }{ }^{\text {a) }} \text {, modified for pregnancy. } \\
\text { Coagulopathy must be detected before loss of sufficient blood to itself account for dilutional or } \\
\text { shock related consumptive coagulopathy. }\end{array}$ & $7(58.3)$ & $5(41.7)$ \\
\hline 3. Clinical onset during labor or within 30 minutes of delivery of placenta & $10(83.3)$ & $2(16.7)$ \\
\hline 4. No fever $\left(\geq 38^{\circ} \mathrm{C}\right)$ during labor & $11(91.7)$ & $1(8.3)$ \\
\hline
\end{tabular}

SABP, systolic arterial blood pressure; SpO2, peripheral capillary oxygen saturation; DIC, disseminated intravascular coagulation; ISTH, International Society on Thrombosis and Hemostasis.

a) Modified ISTH scoring system for overt DIC in pregnancy: platelet count $>100,000 / \mathrm{mL}=0,<100,000 / \mathrm{mL}=1,<50,000=2$. Prolonged prothrombin time or international normalized ratio: $<25 \%$ increase $=0,25 \%-50 \%$ increase $=1,>50 \%$ increase $=2$. Fibrinogen level: $>2 \mathrm{~g} / \mathrm{L}=0,<2 \mathrm{~g} / \mathrm{L}=1$. Score $\geq 3$ is compatible with overt DIC in pregnancy. 


\title{
Obstetrics \& Gynecology Science
}

\author{
Vol. 64, No. 2, 2021
}

tact maternal survival was $41.7 \%$. Among the 5 intrapartum cases, no case of neonatal death was found, and 1 neonate (case number 1) had neurologic disability due to hypoxic ischemic encephalopathy.

Table 3 summarizes the disagreement between AFE cases and the 4 criteria proposed by the SMFM. Overall, $66.7 \%$ of the cases in our study did not satisfy all 4 diagnostic criteria. The most unsatisfied criterion was the documentation of DIC before the loss of sufficient blood (41.7\%). In detail, the initial assessment by blood test, including the coagulation test, was not available in 4 cases since these tests were performed after the loss of sufficient blood (case number 1 , $6,11,12$ ). Documentation of DIC, which was defined as a modified ISTH score of 3 or more, was not evident in 1 case (case number 5). In addition, the criteria for sudden onset of cardiorespiratory arrest or both hypotension and respiratory compromise was not met in $16.7 \%$ of the cases (case number 4 and 8). The criterion for the strict onset time, i.e., within 30 min of delivery, was also not met by $16.7 \%$ of the cases, in which the onset time was within 1-2 hours after placenta delivery (case number 4 and 6). One patient had fever (temperature $\geq 38^{\circ} \mathrm{C}$ ) during labor (case number 3 ).

Table 4 compares the maternal outcomes and management of AFE among the cases satisfying all 4 proposed criteria (group I) and those with at least 1 missing criterion (group II). There was no difference in maternal mortality, $25 \%$ in group I and $50 \%$ in group || $(P=0.576)$. The incidence of massive bleeding, the amount of total transfusion, the rate of ECMO, hysterectomy, and ICU care also showed no differences between the 2 groups.

\section{Discussion}

Our data showed that two-thirds of AFE cases, clinically confirmed by MFM specialists, did not satisfy all 4 criteria proposed by the SMFM. The documentation of DIC was the most unsatisfied criterion, where $41.7 \%$ of AFE cases missed it. In addition, $16.7 \%$ missed the clinical onset time criterion of within 30 minutes of delivery. Given these results, our study is in line with a French national study, including 36 cases of maternal mortality due to AFE, which showed that $41.7 \%$ of the cases did not satisfy at least 1 diagnostic criterion [8]. In their study, initial laboratory confirmation of DIC was also the most commonly unsatisfied criterion (28.9\%), and the clinical onset time criterion of within 30 minutes after delivery was missed in 5.6\% of women [8]. Moreover, according to a recent study by the Amniotic Fluid Embolism

Table 4. Comparison of maternal outcomes and management of amniotic fluid embolism (AFE) between cases satisfying all 4 proposed criteria and cases with at least one missing criterion

\begin{tabular}{|c|c|c|c|c|}
\hline Characteristics & $\begin{array}{l}\text { Total } \\
(n=12)\end{array}$ & $\begin{array}{l}\text { All } 4 \text { diagnostic criteria } \\
(\text { Group I, n=4) })^{\mathrm{a})}\end{array}$ & $\begin{array}{l}\text { At least one missing } \\
\text { criterion } \\
\text { (Group II, n=8) }\end{array}$ & $P$-value \\
\hline Maternal death & $5(41.7)$ & $1(25.0)$ & $4(50.0)$ & 0.576 \\
\hline Massive bleeding (>1,000 mL) & $9(90.0)$ & $3(75.0)$ & $6(100.0)$ & 0.400 \\
\hline Total transfusion & $36.0(27.8-47.5)$ & $44.0(33.8-115.8)$ & $34.5(25.5-43.0)$ & 0.349 \\
\hline $\mathrm{pRBC}$ & $9.0(7.8-16.5)$ & $14.0(8.8-41.2)$ & $8.5(6.5-14.5)$ & 0.173 \\
\hline FFP & $9.5(6.8-21.2)$ & $9.5(8.2-23.8)$ & $12.0(5.8-21.2)$ & 0.734 \\
\hline Platelets & $5.5(3.0-10.5)$ & $9.5(6.0-42.5)$ & $5.0(2.2-7.0)$ & 0.200 \\
\hline Cryoprecipitate & $0.0(0.0-3.8)$ & $3.0(0.0-10.0)$ & $0.0(0.0-2.2)$ & 0.570 \\
\hline SDP & $0.0(0.0-1.0)$ & $0.0(0.0-1.0)$ & $0.0(0.0-1.0)$ & 0.911 \\
\hline ECMO & $4(33.3)$ & $1(25.0)$ & $3(37.5)$ & 1.000 \\
\hline Hysterectomy & $3(25.0)$ & $1(25.0)$ & $2(25.0)$ & 1.000 \\
\hline ICU care & $7(58.3)$ & $3(75.0)$ & $4(50.0)$ & 0.576 \\
\hline
\end{tabular}

Data presented as number (\%) or median (interquartile range).

pRBC, packed red blood cells; FFP, fresh frozen plasma; SDP, single donor platelets; ECMO, extracorporeal membrane oxygenation; ICU, intensive care unit.

${ }^{\text {a) }}$ Case number of group I was 2, 7, 9, and 10 as shown in Table 2. 


\section{Obstetrics \& Gynecology Science}

Jin-ha Kim, et al. Diagnosis of amniotic fluid embolism

Registry in the Unites States, $21 \%$ of clinically diagnosed AFEs did not meet the criteria by the SMFM for use in research [9]. Collectively, these studies indicate that routine adoption of the recent diagnostic criteria by the SMFM for research purpose in clinical practice is too strict to diagnose all AFE cases, especially in terms of early documentation of DIC and the onset time, possibly resulting concerns from delayed clinical diagnosis and management.

To meet the SMFM criteria, all DIC tests should have been performed before the loss of sufficient blood. However, in dire situations, such as sudden cardiorespiratory arrest or massive bleeding, it is the main priority of the clinicians to immediately perform resuscitative procedures, making it difficult to promptly conduct initial blood tests. Such a scenario is particularly common in local hospitals where human resources and medical facilities are limited. Although coagulopathy is recognized as the main criterion for AFE, the requirement of early laboratory documentation of coagulopathy proposed by the SMFM can lead to exclusion of some clinical AFE cases during diagnosis. In fact, in our study, 4 out of 7 transferred cases had late documentations of DIC after arrival at the referral hospitals. Our results also showed that cases with at least 1 missing criterion manifested fatal maternal outcomes regarding maternal mortality and the amount of transfusion, similar to cases satisfying all 4 criteria. In this regard, we support the French national study that suggested extending the early documentation of DIC criteria of the SMFM to early clinical coagulopathy with bleeding [8].

Typical symptoms and signs of AFE include rapid deterioration of maternal condition, sudden onset of cardiac arrest or hypotension, dyspnea, and cyanosis. However, consumption coagulopathy without cardiopulmonary arrest was recently proposed as an incomplete form of AFE [15]. Intriguingly, in our study, there were 2 cases (case number 4 and 8 ) that manifested symptoms of clinical coagulopathy and neurologic signs without cardiorespiratory compromise. In fact, Kanayama and Tamura [16] named these atypical cases as DIC-type AFE, caused by a localized anaphylactoid reaction in the uterus. This condition is characterized by the presence of severe coagulopathy and incoagulable bleeding without cardiopulmonary arrest at the initial stage, followed by massive blood loss during delivery at a later stage. The characteristics of autopsy findings included uterine atony (a large, edematous uterus) observed macroscopically and amniotic components observed microscopically in the uterine vessels
[16]. Histological findings showed the presence of inflammatory cells and mast cells within the myometrium, proposed as "postpartum acute myometritis" [17]. It was speculated that the local flow of amniotic fluid into the uterine tissues may cause an anaphylactoid reaction in the uterus, leading to an edematous uterus and a loss of synchronized contraction of the myometrium.

According to the analysis of the Maternal Mortality Evaluation Committee in Japan from January 2010 to November 2012, 21 of 75 maternal deaths were caused by AFE [16]. Of these 21 cases, 10 suffered cardiopulmonary collapse and 11 had atonic bleeding/DIC. Based on these data, Japanese obstetricians recognize cases with maternal hemorrhage as the first presenting feature of AFE even with no evidence of cardiorespiratory compromise [6], unlike the criteria from the United States and United Kingdom [7,18]. Importantly, it was indicated that inclusion of the atypical DIC-type in the AFE criteria enables prompt recognition and treatment of AFE, contributing to decreasing maternal mortality rate in Japan [19].

In our postpartum cases of AFE, 5 of 7 cases showed the first signs and symptoms within 30 minutes of delivery, while 2 cases manifested clinical onset between 1 and 2 hours after delivery. So far, several different criteria for the clinical onset time of AFE have been suggested. In Japan, clinical onset was defined as "symptoms appearing within 12 hours of delivery" [6]. The International Network of Obstetric Survey System (INOSS), a multi-country collaboration in Europe, defined it as "symptoms appearing within 6 hours after labor, delivery, or ruptured membranes" [20]. Current SMFM criteria defined it as "within 30 minutes of delivery of the placenta," presenting the strictest time interval in postpartum AFE cases.

Of note, in our study, overall $66.7 \%$ of the cases showed neurologic symptoms and 50\% (case number 3, 4, 7, 8, 9, 12) showed neurologic symptoms as the first sign, which was the most common symptom. Symptoms of restlessness, confusion, agitation, or numbness have traditionally been considered to support AFE diagnosis [8]. The United Kingdom diagnostic criteria include neurologic signs, such as restlessness, numbness, agitation, and tingling [5]. Therefore, there is a possibility that clinically typical AFE cases may have been excluded when the SMFM criteria for research purpose were applied.

Our data showed that the chance of intact maternal sur- 


\title{
Obstetrics \& Gynecology Science
}

\author{
Vol. 64, No. 2, 2021
}

vival in AFE was less than half (41.7\%). According to Korean statistics from 2018, direct maternal death accounted for $62.2 \%$ of all maternal deaths. Obstetric embolism, related to the increase in maternal age, was an important cause of maternal mortality in $21.6 \%$ cases [3]. The average childbearing age in Korea increased from 30.3 years in 2008 to 32.8 years in 2018. The proportion of elderly mothers over the age of 35 years steadily increased from $14.3 \%$ in 2008 to $31.8 \%$ in 2018 [21]. The incidence of AFE is rising possibly due to an increase in maternal age, which is one of the most important risk factors of AFE [22]. In fact, it was noted that the risk of AFE increased stepwise according to maternal age (adjusted odds ratio $[\mathrm{aOR}], 1.12 ; 95 \%$ confidence interval [Cl], 1.081.15; $P<0.001$ for every 1-year increase in age), and its risk was 2.4 times higher for mothers over 35 years of age (aOR, 2.40; 95\% Cl, 1.71-3.37) [23]. According to a study by Chung et al. [24] comparing mean maternal age at childbirth in the same year (2012), maternal age in Korea (31.6 years) was markedly higher than that in the United States (25.8 years) and England (29.8 years). In addition, the percentage of mothers aged 30-34 years in Korea was $49.4 \%$, which was higher than that in the United States (25.6\%) and England (29.3\%) [24]. Taken together, it is highly possible that the incidence of AFE will also increase in Korea, considering the increase in maternal age.

There are some limitations to this study. Since this study is a kind of case series rather than a study from a complete retrospective cohort, some cases of AFE may have been missed out during the study period. At the same time, 7 out of 12 cases in our study were immediately transferred to referral hospitals; thus, there could have been a potential selection bias and less generalizability, resulting in underestimation of maternal mortality of AFE and precluding the assessment of the exact prevalence of AFE. Meanwhile, all 12 cases, including 7 cases transferred from local hospitals, were discussed with the MFM specialists during inclusion and categorization for consensus. For example, during the confirmation of AFE, primary uterine atony; consumptive coagulopathy/DIC because of evident etiologies, such as abnormal placentation, trauma during labor, and severe preeclampsia/eclampsia; and other types of medical critical illness were excluded. Our case series still presented a wide spectrum of clinical characteristics, including initial symptoms, laboratory findings, the amount of transfusion, and the management of individual cases, which could provide indirect experiences for readers and ultimately aid in the understanding of such rare yet fatal diseases.

Our study also poses a limitation in evaluating the diagnostic performance of autopsy in cases of maternal mortality. In our study, only 1 out of the 5 maternal mortality cases underwent an autopsy and was finally diagnosed as AFE. Conventional fetal squamous cells in the maternal pulmonary artery are no longer considered diagnostic of AFE [1] because several reports have documented identical pathologic findings in pregnant women, unrelated to AFE $[25,26]$. Recently, several serum markers, including $\beta$-tryptase, decreased C3 and C4 levels, and lower $\mathrm{C} 1$ esterase inhibitors, have been proposed as diagnostic tests for AFE, although they are still under investigation $[27,28]$. In addition, in mortality cases, some histopathological tests for C3a and tryptase degranulation in the uterus and lungs have been suggested as promising tools for diagnosing AFE [29]. Collectively, these data indicate that no single laboratory or clinical finding can be used to diagnose or exclude AFE [29]. Such variability is caused by the antigenic variability of fetal antigens and individual immune responses similar to other anaphylactoid reactions [30].

In conclusion, there may be some discrepancy between the clinical diagnosis of AFE and recent diagnostic criteria proposed by the SMFM for research purposes. Furthermore, this review of case series demonstrated that there is a need to perform DIC tests immediately if clinical symptoms of AFE such as sudden cardiovascular collapse or unexplained neurologic symptoms occur, followed by aggressive supportive management, including the correction of DIC [31]. We also argue that there is an urgent need to collect clinical information on AFE cases from local and referral hospitals and build the Korean National AFE Registry. Finally, we suggest using the questionnaire form used in this study (Supplementary Data 1) to collect detailed clinical information on suspected AFE anonymously and thoroughly.

\section{Conflict of interest}

No potential conflict of interest relevant to this article was reported.

\section{Ethical approval}

The study was approved by the Institutional Review Board 


\section{Obstetrics \& Gynecology Science}

Jin-ha Kim, et al. Diagnosis of amniotic fluid embolism

of Samsung Medical Center (IRB No. 2020-06-028-002) and performed in accordance with the principles of the Declaration of Helsinki.

\section{Patient consent}

None.

\section{Funding information}

None.

\section{Supplementary material}

Supplementary Data 1 associated with this article can be found online at https://doi.org/10.5468/ogs.20195.

\section{References}

1. Clark SL. Amniotic fluid embolism. Obstet Gynecol 2014;123:337-48.

2. Knight $M$, Tuffnell $D$, Brocklehurst $P$, Spark $P$, Kurinczuk JJ; UK Obstetric Surveillance System. Incidence and risk factors for amniotic-fluid embolism. Obstet Gynecol 2010;115:910-7.

3. Statistics Korea. Cause of maternal death. In: Report No. 11-1240000-000652-10. Infant, maternal and perinatal mortality statistics 2017. Daejeon: Statistics Korea; 2018. p.26.

4. Australasian Maternity Outcomes Surveillance System. Amniotic fluid embolism. Sydney: Australasian Maternity Outcomes Surveillance System; c2020 [cited 2016 Jun 9]. Available from: http://www.amoss.com. au/?q1/4content/amniotic-fluid-embolism-afe.

5. UK Obstetric Surveillance System. Amniotic fluid embolism. Oxford: University of Oxford, Old Road Campus; c2020 [cited 2016 Jun 9]. Available from: https://www. npeu.ox.ac.uk/ukoss/current-surveillance/amf.

6. Hasegawa J, Sekizawa A, Tanaka H, Katsuragi S, Osato K, Murakoshi T, et al. Current status of pregnancy-related maternal mortality in Japan: a report from the Mater- nal Death Exploratory Committee in Japan. BMJ Open 2016;6:e010304.

7. Clark SL, Romero R, Dildy GA, Callaghan WM, Smiley RM, Bracey AW, et al. Proposed diagnostic criteria for the case definition of amniotic fluid embolism in research studies. Am J Obstet Gynecol 2016;215:408-12.

8. Bonnet MP, Zlotnik D, Saucedo M, Chassard D, BouvierColle MH, Deneux-Tharaux $C$, et al. Maternal death due to amniotic fluid embolism: a national study in France. Anesth Analg 2018;126:175-82.

9. Stafford IA, Moaddab A, Dildy GA, Klassen M, Belfort $M A$, Romero $R$, et al. Evaluation of proposed criteria for research reporting of amniotic fluid embolism. Am J Obstet Gynecol 2019;220:285-7.

10. Korea Supreme Court. Sentencing of 96da43164 (1997).

11. Busan District Court. Sentencing of 2009gahab15735 (2012).

12. Uijeongbu District Court. Sentencing of 2010 gahab5837 (2010).

13. Downes KL, Grantz KL, Shenassa ED. Maternal, labor, delivery, and perinatal outcomes associated with placental abruption: a systematic review. Am J Perinatol 2017;34:935-57.

14. Abenhaim HA, Azoulay L, Kramer MS, Leduc L. Incidence and risk factors of amniotic fluid embolisms: a population-based study on 3 million births in the United States. Am J Obstet Gynecol 2008;199:49.e1-8.

15. Hasegawa A, Murakoshi T, Otsuki Y, Torii Y. Clinical course of disseminated intravascular coagulopathy-type amniotic fluid embolism: a report of three cases. J Obstet Gynaecol Res 2016;42:1881-5.

16. Kanayama N, Tamura N. Amniotic fluid embolism: pathophysiology and new strategies for management. J Obstet Gynaecol Res 2014;40:1507-17.

17. Tamura N, Farhana M, Oda T, Itoh H, Kanayama N. Amniotic fluid embolism: pathophysiology from the perspective of pathology. J Obstet Gynaecol Res 2017;43:627-32.

18. Tuffnell $D$, Knight $M$, Plaat F. Amniotic fluid embolism an update. Anaesthesia 2011;66:3-6.

19. Kobayashi H, Akasaka J, Naruse K, Sado T, Tsunemi T, Niiro $\mathrm{E}$, et al. Comparison of the different definition criteria for the diagnosis of amniotic fluid embolism. J Clin Diagn Res 2017;11:QC18-21.

20. Schaap T, Bloemenkamp K, Deneux-Tharaux C, Knight 


\section{Obstetrics \& Gynecology Science}

Vol. 64, No. 2, 2021

M, Langhoff-Roos J, Sullivan E, et al. INOSS. Defining definitions: a Delphi study to develop a core outcome set for conditions of severe maternal morbidity. BJOG 2019;126:394-401.

21. Statistics Korea. Average age for childbirth of maternal. In: Birth statistics for 2018. Daejeon: Statistics Korea; 2018. p.7.

22. Oh SY. The collapse of infrastructure for childbirth: causes and consequences. J Korean Med Assoc 2016;59:417-23.

23. Fitzpatrick KE, van den Akker T, Bloemenkamp KW, Deneux-Tharaux C, Kristufkova A, Li Z, et al. Risk factors, management, and outcomes of amniotic fluid embolism: A multicountry, population-based cohort and nested case-control study. PLoS Med 2019;16:e1002962.

24. Chung SH, Seol HJ, Choi YS, Oh SY, Kim A, Bae CW. Changes in the cesarean section rate in Korea (19822012) and a review of the associated factors. J Korean Med Sci 2014;29:1341-52.

25. Clark SL. New concepts of amniotic fluid embolism: a review. Obstet Gynecol Surv 1990;45:360-8.

26. Haftel A, Chowdhury YS. Amniotic fluid embolism. In:
StatPearls. Treasure Island (FL): StatPearls Publishing; 2020. [cited 2020 Jul 2]. Available from: https://www. ncbi.nlm.nih.gov/books/NBK559107/.

27. Busardò FP, Frati P, Zaami S, Fineschi V. Amniotic fluid embolism pathophysiology suggests the new diagnostic armamentarium: $\beta$-tryptase and complement fractions C3-C4 are the indispensable working tools. Int J Mol Sci 2015;16:6557-70.

28. Benson MD. A hypothesis regarding complement activation and amniotic fluid embolism. Med Hypotheses 2007;68:1019-25.

29. Fineschi V, Riezzo I, Cantatore S, Pomara C, Turillazzi E, Neri M. Complement C3a expression and tryptase degranulation as promising histopathological tests for diagnosing fatal amniotic fluid embolism. Virchows Arch 2009;454:283-90.

30. Benson MD. Nonfatal amniotic fluid embolism. Three possible cases and a new clinical definition. Arch Fam Med 1993;2:989-94.

31. Sultan P, Seligman K, Carvalho B. Amniotic fluid embolism: update and review. Curr Opin Anaesthesiol 2016;29:288-96. 